\title{
Differential cross sections for neutron-proton scattering in the region of the $d^{*}(\mathbf{2 3 8 0})$ dibaryon resonance
}

P. Adlarson, ${ }^{1}$ W. Augustyniak, ${ }^{2}$ W. Bardan, ${ }^{3}$ M. Bashkanov, ${ }^{4}$ F. S. Bergmann, ${ }^{5}$ M. Berłowski,${ }^{6}$ H. Bhatt, ${ }^{7}$ M. Büscher,,${ }^{8,9}$

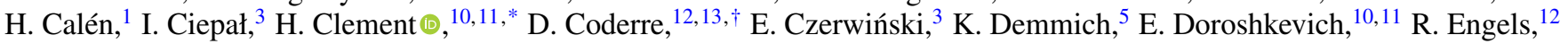
A. Erven, ${ }^{14}$ W. Erven,${ }^{14}$ W. Eyrich, ${ }^{15}$ P. Fedorets,,${ }^{12,16}$ K. Föhl, ${ }^{17}$ K. Fransson, ${ }^{1}$ F. Goldenbaum, ${ }^{12}$ P. Goslawski, ${ }^{5}$

A. Goswami, ${ }^{12,18}$ K. Grigoryev, ${ }^{19,20}$ C.-O. Gullström, ${ }^{1}$ F. Hauenstein,,${ }^{15}$ L. Heijkenskjöld, ${ }^{1}$ V. Hejny, ${ }^{12}$ M. Hodana, ${ }^{3}$ B. Höistad, ${ }^{1}$ N. Hüsken, ${ }^{5}$ A. Jany, ${ }^{3}$ B. R. Jany, ${ }^{3}$ T. Johansson, ${ }^{1}$ B. Kamys, ${ }^{3}$ G. Kemmerling, ${ }^{14}$ F. A. Khan, ${ }^{12}$ A. Khoukaz,${ }^{5}$ D. A. Kirillov, ${ }^{21}$ S. Kistryn, ${ }^{3}$ H. Kleines,${ }^{14}$ B. Kłos, ${ }^{22}$ M. Krapp, ${ }^{15}$ W. Krzemień, ${ }^{23}$ P. Kulessa, ${ }^{24}$ A. Kupść,,${ }^{1,6}$ K. Lalwani, ${ }^{7}$ D. Lersch, ${ }^{12}$ B. Lorentz, ${ }^{12}$ A. Magiera, ${ }^{3}$ R. Maier, ${ }^{12}$ P. Marciniewski, ${ }^{1}$ B. Mariański, ${ }^{2}$ M. Mikirtychiants, ${ }^{12,13,20}$ H.-P. Morsch, ${ }^{2}$ P. Moskal,${ }^{3}$ H. Ohm,${ }^{12}$ I. Ozerianska, ${ }^{3}$ E. Perez del Rio,${ }^{10,11, \S}$ N. M. Piskunov,${ }^{21}$ P. Podkopał,${ }^{3}$ D. Prasuhn, ${ }^{12}$ A. Pricking,,${ }^{10,11}$ D. Pszczel, ${ }^{1,6}$ K. Pysz, ${ }^{24}$ A. Pyszniak, ${ }^{1,3}$ C. F. Redmer, ${ }^{1, \|}$ J. Ritman,,${ }^{12,13}$ A. Roy, ${ }^{18}$ Z. Rudy, ${ }^{3}$ S. Sawant,,${ }^{72}$ S. Schadmand, ${ }^{12}$ T. Sefzick, ${ }^{12}$ V. Serdyuk, ${ }^{12,25}$ R. Siudak, ${ }^{24}$ T. Skorodko, ${ }^{10,11,26}$ M. Skurzok, ${ }^{3}$ J. Smyrski, ${ }^{3}$ V. Sopov, ${ }^{16}$ R. Stassen, ${ }^{12}$ J. Stepaniak, ${ }^{6}$ E. Stephan, ${ }^{22}$ G. Sterzenbach, ${ }^{12}$ H. Stockhorst, ${ }^{12}$ H. Ströher,${ }^{12}$ A. Szczurek, ${ }^{24}$ A. Täschner, ${ }^{5}$ A. Trzciński, ${ }^{2}$ R. Varma, ${ }^{7}$ M. Wolke, ${ }^{1}$ A. Wrońska, ${ }^{3}$ P. Wüstner, ${ }^{14}$ P. Wurm,,${ }^{12}$ A. Yamamoto, ${ }^{27}$ L. Yurev,,${ }^{25, \mathbb{I}}$ J. Zabierowski, ${ }^{28}$ M. J. Zieliński, ${ }^{3}$ A. Zink, ${ }^{15}$ J. Złomańczuk, ${ }^{1}$ P. Żuprański, ${ }^{2}$ and M. Żurek ${ }^{12}$

(WASA-at-COSY Collaboration)

\section{R. L. Workman, ${ }^{29}$ W. J. Briscoe,${ }^{29}$ and I. I. Strakovsky ${ }^{29}$}

(SAID Data Analysis Center)

$$
\text { A. } \check{S} \operatorname{varc}^{30,31}
$$

${ }^{1}$ Division of Nuclear Physics, Department of Physics and Astronomy, Uppsala University, Box 516, 75120 Uppsala, Sweden

${ }^{2}$ Department of Nuclear Physics, National Centre for Nuclear Research, ul. Hoza 69, 00-681 Warsaw, Poland

${ }^{3}$ Institute of Physics, Jagiellonian University, ul. Reymonta 4, 30-059 Kraków, Poland

${ }^{4}$ Department of Physics, University of York, Heslington, York YO10 5DD, United Kingdom

${ }^{5}$ Institut für Kernphysik, Westfälische Wilhelms-Universität Münster, Wilhelm-Klemm-Str. 9, 48149 Münster, Germany

${ }^{6}$ High Energy Physics Department, National Centre for Nuclear Research, ul. Hoza 69, 00-681 Warsaw, Poland

${ }^{7}$ Department of Physics, Indian Institute of Technology Bombay, Powai, Mumbai 400076, Maharashtra, India

${ }^{8}$ Peter Grünberg Institut, Forschungszentrum Jülich, 52425 Jülich, Germany

${ }^{9}$ Institut für Laser- und Plasmaphysik, Heinrich-Heine Universität Düsseldorf, 40225 Düsseldorf, Germany

${ }^{10}$ Physikalisches Institut, Eberhard-Karls-Universität Tübingen, Auf der Morgenstelle 14, 72076 Tübingen, Germany

${ }^{11}$ Kepler Center for Astro and Particle Physics, University of Tübingen, Auf der Morgenstelle 14, 72076 Tübingen, Germany

${ }^{12}$ Institut für Kernphysik, Forschungszentrum Jülich, 52425 Jülich, Germany

${ }^{13}$ Institut für Experimentalphysik I, Ruhr-Universität Bochum, Universitätsstr. 150, 44780 Bochum, Germany

${ }^{14}$ Zentralinstitut für Engineering, Elektronik und Analytik, Forschungszentrum Jülich, 52425 Jülich, Germany

${ }^{15}$ Physikalisches Institut, Friedrich-Alexander-Universität Erlangen-Nürnberg, Erwin-Rommel-Str. 1, 91058 Erlangen, Germany

${ }^{16}$ Institute for Theoretical and Experimental Physics, State Scientific Center of the Russian Federation, Bolshaya Cheremushkinskaya 25, 117218 Moscow, Russia

${ }^{17}$ II Physikalisches Institut, Justus-Liebig-Universität Gießen, Heinrich-Buff-Ring 16, 35392 Giessen, Germany

${ }^{18}$ Department of Physics, Indian Institute of Technology Indore, Khandwa Road, Indore 452017, Madhya Pradesh, India

${ }^{19}$ III Physikalisches Institut B, Physikzentrum, RWTH Aachen, 52056 Aachen, Germany

${ }^{20}$ High Energy Physics Division, Petersburg Nuclear Physics Institute, Orlova Rosha 2, Gatchina, Leningrad District 188300, Russia

${ }^{21}$ Veksler and Baldin Laboratory of High Energiy Physics, Joint Institute for Nuclear Physics, Joliot-Curie 6, 141980 Dubna, Russia

${ }^{22}$ August Chetkowski Institute of Physics, University of Silesia, Uniwersytecka 4, 40-007 Katowice, Poland

${ }^{23}$ High Energy Physics Division, National Centre for Nuclear Research, 05-400 Otwock-wierk, Poland

${ }^{24}$ The Henryk Niewodniczański Institute of Nuclear Physics, Polish Academy of Sciences, 152 Radzikowskiego Street, $31-342$ Kraków, Poland

${ }^{25}$ Dzhelepov Laboratory of Nuclear Problems, Joint Institute for Nuclear Physics, Joliot-Curie 6, 141980 Dubna, Russia

${ }^{26}$ Department of Physics, Tomsk State University, 36 Lenin Avenue, Tomsk, 634050 Russia

${ }^{27}$ High Energy Accelerator Research Organisation KEK, Tsukuba, Ibaraki 305-0801, Japan

${ }^{28}$ Astrophysics Division, National Centre for Nuclear Research, Box 447, 90-950 Łódź, Poland

${ }^{29}$ Data Analysis Center at the Institute for Nuclear Studies, Department of Physics, The George Washington University, Washington, DC 20052, USA

${ }^{30}$ Rudjer Bošković Institute, Bijenička cesta 54, P.O. Box 180, 10002 Zagreb, Croatia

${ }^{31}$ Tesla Biotech, Mandlova 7, 10002 Zagreb, Croatia 


\begin{abstract}
Differential cross sections have been extracted from exclusive and kinematically complete high-statistics measurements of quasifree polarized $\vec{n} p$ scattering performed in the energy region of the $d^{*}(2380)$ dibaryon resonance covering the range of beam energies $T_{n}=0.98-1.29 \mathrm{GeV}(\sqrt{s}=2.32-2.44 \mathrm{GeV})$. The experiment was carried out with the WASA-at-COSY setup having a polarized deuteron beam impinged on the hydrogen

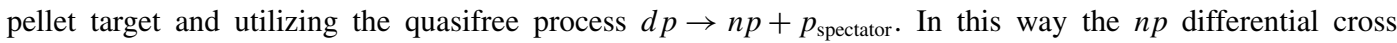
section $\sigma(\Theta)$ was measured over a large angular range. The obtained angular distributions complement the corresponding analyzing power $A_{\mathrm{y}}(\Theta)$ measurements published previously. A SAID partial-wave analysis incorporating the new data strengthens the finding of a resonance pole in the coupled ${ }^{3} D_{3}-{ }^{3} G_{3}$ waves.
\end{abstract}

DOI: 10.1103/PhysRevC.102.015204

\section{INTRODUCTION}

Recently a resonance pole with $I\left(J^{P}\right)=0\left(3^{+}\right)$at $(2380 \pm$ $10-i 40 \pm 5) \mathrm{MeV}$ - denoted $d^{*}(2380)$-was discovered in the coupled ${ }^{3} D_{3}-{ }^{3} G_{3}$ partial waves (PWs) of nucleon-nucleon $(N N)$ scattering by the SAID partial-wave analysis based on the full SAID database and recent analyzing-power data provided by WASA-at-COSY for the laboratory energy range $T_{n}=1.095-1.270 \mathrm{GeV}(\sqrt{s}=2.36-2.43 \mathrm{GeV})[1,2]$. The values for this pole coincide with a pronounced narrow resonance structure previously observed in the total cross section of the basic isoscalar double-pionic fusion reaction $p n \rightarrow$ $d \pi^{0} \pi^{0}[3,4]$ at a mass $M \approx 2370 \mathrm{MeV}$ with a width of $\Gamma \approx$ $70 \mathrm{MeV}$. From the angular distributions spin-parity $J^{P}=3^{+}$ was deduced [4]. Additional evidence for $d^{*}$ (2380) has been found recently in the $p n \rightarrow d \pi^{+} \pi^{-}[5], p n \rightarrow p p \pi^{0} \pi^{-}[6]$, $p n \rightarrow p n \pi^{0} \pi^{0}$ [7], and $p n \rightarrow p n \pi^{+} \pi^{-}$[8,9] reactions. In measurements of the isoscalar single-pion production cross section no significant decay of this resonance into the isoscalar $[N N \pi]_{I=0}$ channel has been observed-providing a small upper limit [10]. Thus all branchings of this resonance into the hadronic decay channels $N N, N N \pi$, and $N N \pi \pi$ have been extracted $[10,11]$. They agree with the decay of a deeply bound $\Delta \Delta$ system $[11,12]$, possibly accompanied by a small admixture of a $D_{12} \pi$ configuration [13], where $D_{12}$ denotes the $I\left(J^{P}\right)=1\left(2^{+}\right)$resonance structure near the $\Delta N$ threshold.

\footnotetext{
*clement@pit.physik.uni-tuebingen.de

'Present address: Albert Einstein Center for Fundamental Physics, University of Bern, Sidlerstrasse 5, 3012 Bern, Switzerland.

${ }^{\ddagger}$ Present address: Department of Physics and Astrophysics, University of Delhi, Delhi 110007, India.

${ }^{\S}$ Present address: Dipartimento di Fisica dell Universita Sapienza, Roma, Italy and INFN Sezione di Roma, Roma, Italy.

"Present address: Institut für Kernphysik, Johannes GutenbergUniversität Mainz, Johann-Joachim-Becher Weg 45, 55128 Mainz, Germany.

"Present address: Department of Physics and Astronomy, University of Sheffield, Hounsfield Road, Sheffield S3 7RH, United Kingdom.
}

Published by the American Physical Society under the terms of the Creative Commons Attribution 4.0 International license. Further distribution of this work must maintain attribution to the author(s) and the published article's title, journal citation, and DOI. Funded by $S C O A P^{3}$.
For a discussion of the latter see, e.g., Ref. [12]. Recently also suggestive evidence for photoexitation of $d^{*}(2380)$ has been found in measurements of the $\gamma d \rightarrow d \pi^{0} \pi^{0}$ reaction [14,15].

In addition to the ample evidence for the dibaryon resonance $d^{*}(2380)$ the estasblishment of its resonance pole in $n p$ scattering certainly is of particular importance. This finding is solely based on the analyzing power data provided by WASAat-COSY. Hence it appears highly desirable to supplement this database in the region of the $d^{*}(2380)$ resonance by comprehensive differential cross-section data, since previous measurements mainly covered either just the very forwardangle [16] or just the backward-angle [17] region.

\section{EXPERIMENT}

For the extraction of the differential cross sections in the region of the $d^{*}(2380)$ resonance we use the same data set as exploited before for extraction of the analyzing powers $[1,2]$. For this purpose the $n p$ elastic scattering was measured in the quasifree mode with the WASA detector including a hydrogen pellet target $[19,20]$ at COSY (Forschungsztentrum Jülich, Germany) and using a polarized deuteron beam with an energy of $T_{d}=2.27 \mathrm{GeV}$. Thus the full energy range of the conjectured resonance was covered. Note that we observe here the quasifree scattering process $d p \rightarrow n p+p_{\text {spectator }}$ in inverse kinematics, which allows the detection of the fast spectator proton in the forward detector of WASA.

Since we deal here with events originating from channels with large cross sections, the trigger was set to at least one hit in the first layer of the forward-range hodoscope. For the case of quasifree $n p$ scattering this defines two event classes, with each of them having the spectator proton detected in the forward detector:

(i) scattered proton and scattered neutron both detected in the central detector covering the neutron angle region $40^{\circ}<\Theta_{n}^{\text {c.m. }}<125^{\circ}$; and

(ii) scattered proton detected in the forward detector with the scattered neutron being unmeasured, thus covering $132^{\circ}<\Theta_{n}^{\text {c.m. }}<145^{\circ}$.

In this way a large range of neutron scattering angles could be covered.

For each selected event one neutral hit in the central detector was required. The $p n$ elastic events have been identified 
by using the kinematic constraints for the opening angle and planarity.

By use of the inverse kinematics the spectator proton is part of the beam particle, the deuteron. Hence the spectator is very fast and this allows its detection in the forward detector. By reconstruction of its kinetic energy and its direction the full four-momentum of the spectator proton has been determined. Similarly the four-momentum of the actively scattered proton has been obtained from its track information in either the forward or the central detector (in the latter case the energy information was not retrieved).

Since the neutron was detected by a hit in the calorimeter [composed of $1012 \mathrm{CsI}(\mathrm{Na})$ crystals] of the central detectorassociated with no hit in the preceding plastic scintillator barrel-only its directional information was obtained. In the subsequent kinematical fit the full event could be reconstructed with two overconstraints in the case of the first event class and with three overconstraints in the case of the second event class.

As noted above, we utilize here data that were obtained by use of a polarized beam for the determination of analyzing powers. Hence, in order not to distort the beam polarization, the magnetic field of the solenoid in the central detector was switched off in that beamtime.

Whereas in analyzing-power measurements, detector efficiencies cancel, the determination of differential cross sections heavily depends on a precise knowledge of detector efficiencies. The latter have been determined by comprehensive Monte Carlo simulations of the WASA detector performance and their cross-check against calibration data.

The momentum distribution of the observed spectator proton in the elastic $n p$ scattering process is plotted in Fig. 1 in the deuteron rest frame and compared with Monte Carlo simulations of the proton momentum distribution in the deuteron. In these simulations, the deuteron wave function of the $\mathrm{CD}$ Bonn potential [21] has been used. Because of the beam pipe, ejectiles can only be detected in the forward detector for laboratory angles larger than $2.5^{\circ}$. In order to assure a quasifree process we omit events with spectator momenta larger than $0.18 \mathrm{GeV} / c$ (in the deuteron rest system) from the subsequent analysis - similar to what was done in previous work [4-6].

The absolute normalization of our data was obtained by normalizing our data at $T_{p}=1.109 \mathrm{GeV}$ to the back-angle data of Bizard et al. [17]. For a cross-check of the absolute normalization of the $n p$ scattering data we analyzed the $d p \rightarrow$ $n p \pi^{0}+p_{\text {spectator }}$ reaction, which had been taken in parallel and with the same trigger. Since there are no high-quality data for the $n p \pi^{0}$ channel at the energy of interest here, we have used the following isospin relation for the total cross sections [18]:

$$
\begin{aligned}
2 \sigma\left(n p \rightarrow n p \pi^{0}\right)= & \sigma\left(p p \rightarrow p n \pi^{+}\right)+2 \sigma\left(n p \rightarrow p p \pi^{-}\right) \\
& -2 \sigma\left(p p \rightarrow p p \pi^{0}\right) .
\end{aligned}
$$

Using the values $17 \pm 2.2 \mathrm{mb}[8], 2.5 \pm 0.2 \mathrm{mb}$, and $4.0 \pm 0.3 \mathrm{mb}$ [10] for the total cross sections of the $p p \rightarrow$ $p n \pi^{+}, n p \rightarrow p p \pi^{-}$, and $p p \rightarrow p p \pi^{0}$ reactions, respectively, we arrive at a total cross section of $6 \pm 1 \mathrm{mb}$ for the $n p \rightarrow$ $n p \pi^{0}$ reaction. Using the absolute normalization as obtained

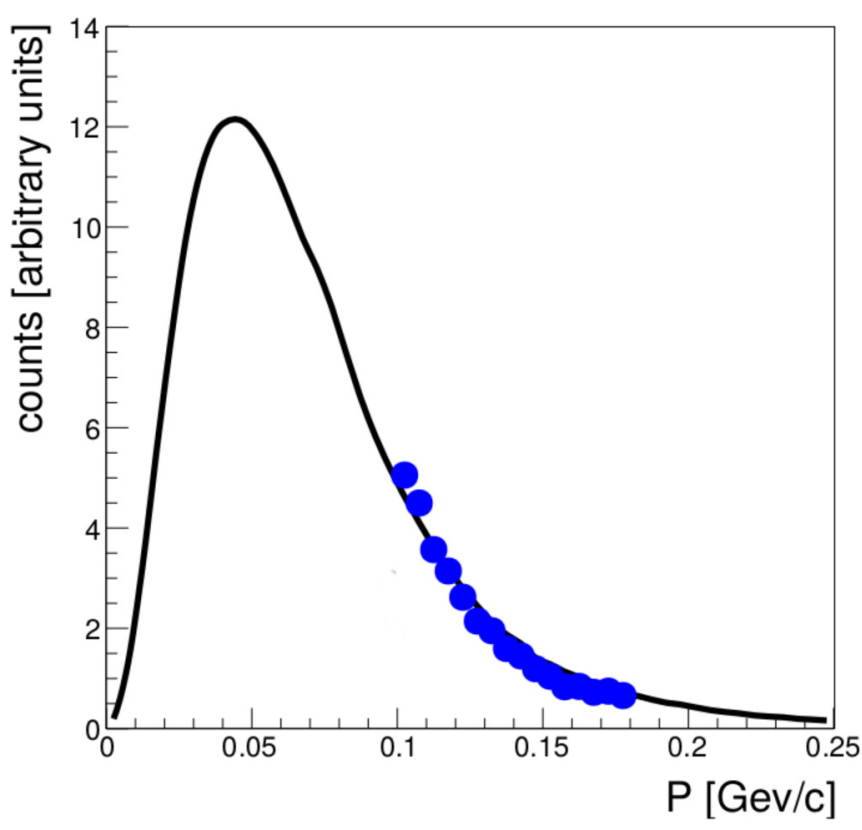

FIG. 1. Distribution of the spectator proton momenta $P$ (in the deuteron rest frame) in the $d p \rightarrow p n+p_{\text {spectator }}$ reaction. Data are represented by filled circles. The solid line shows the expected distribution for the quasifree process based on the CD Bonn potential [21] deuteron wave function. For the data analysis only events with spectator momenta $P<0.18 \mathrm{GeV} / c$ have been used.

from the adjustment of our $n p$ data to those of Bizard et al., we arrive at $7 \mathrm{mb}$ for the $n p \rightarrow n p \pi^{0}$ reaction-in good agreement with the value obtained from the isospin relation.

\section{EXPERIMENTAL RESULTS}

Due to the Fermi motion of the nucleons bound in the beam deuteron, the measurement of the quasifree $n p$ scattering process covers a range of energies in the $n p$ system. Meaningful statistics could be collected for the range of $n p$ centerof-mass energies $2.32<\sqrt{s}<2.44 \mathrm{GeV}$, corresponding to $T_{n}=0.98-1.29 \mathrm{GeV}$.

By taking the measured spectator four-momentum into account and reconstructing in this way the effective $\sqrt{s}$ for each event, we obtain angular distributions for six $\sqrt{s}$ bins as shown in Fig. 2. Our data agree well with previous experimental results from Saturne for backward [17] angles in the overlap region. Where overlapping our results are also in reasonable agreement with the old Birmingham data [22], which were discarded in previous SAID analyses, though they were taken over nearly the full angular range at $T_{p}=0.991 \mathrm{GeV}$. Our data are also in good agreement with the old Berkeley data taken at $T_{p}=1.243 \mathrm{GeV}$ over the full forward angular range [23].

\section{COMPARISON TO EXISTING PARTIAL-WAVE SOLUTIONS}

In Fig. 2 the data are compared to recently obtained GWU/SAID partial-wave solutions. The dotted lines resemble the solution SP07 [24,25], which is based on $N N$ 

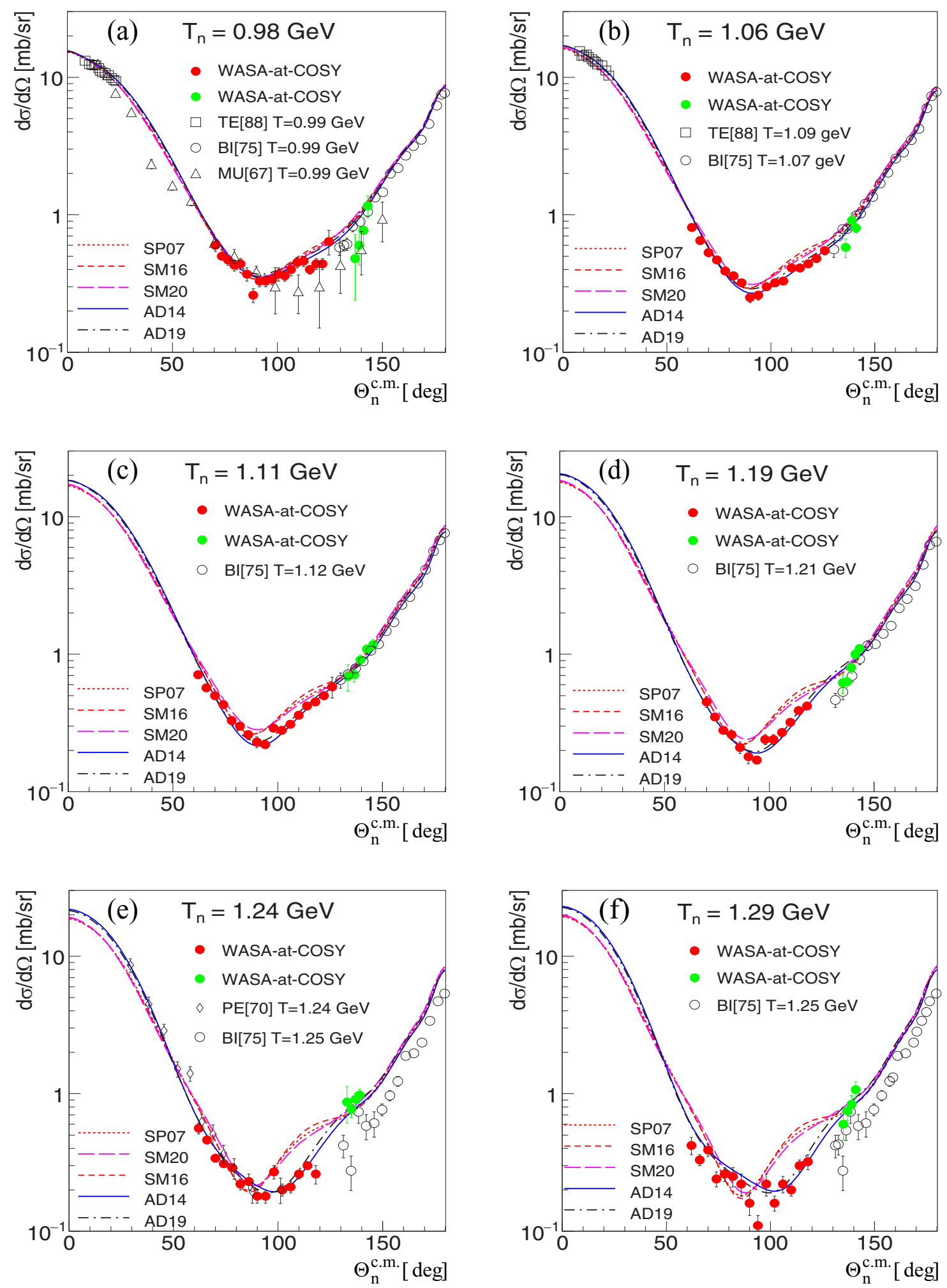

FIG. 2. Differential cross sections for elastic $n p$ scattering at $T_{n}=0.98,1.06,1.11,1.19,1.24$, and $1.29 \mathrm{GeV}$, corresponding to $\sqrt{s}=$ $2.32,2.35,2.37,2.40,2.42$, and $2.44 \mathrm{GeV}$. Filled symbols denote results from this work taking into account the spectator four-momentum information. Open symbols refer to previous measurements: for "TE[88]" see Ref. [16], for "BI[75]" see Ref. [17], for "MU[67]” see Ref. [22], and for "PE[70]" see Ref. [23]. The drawn curves represent various GWU/SAID solutions discussed in the text. 

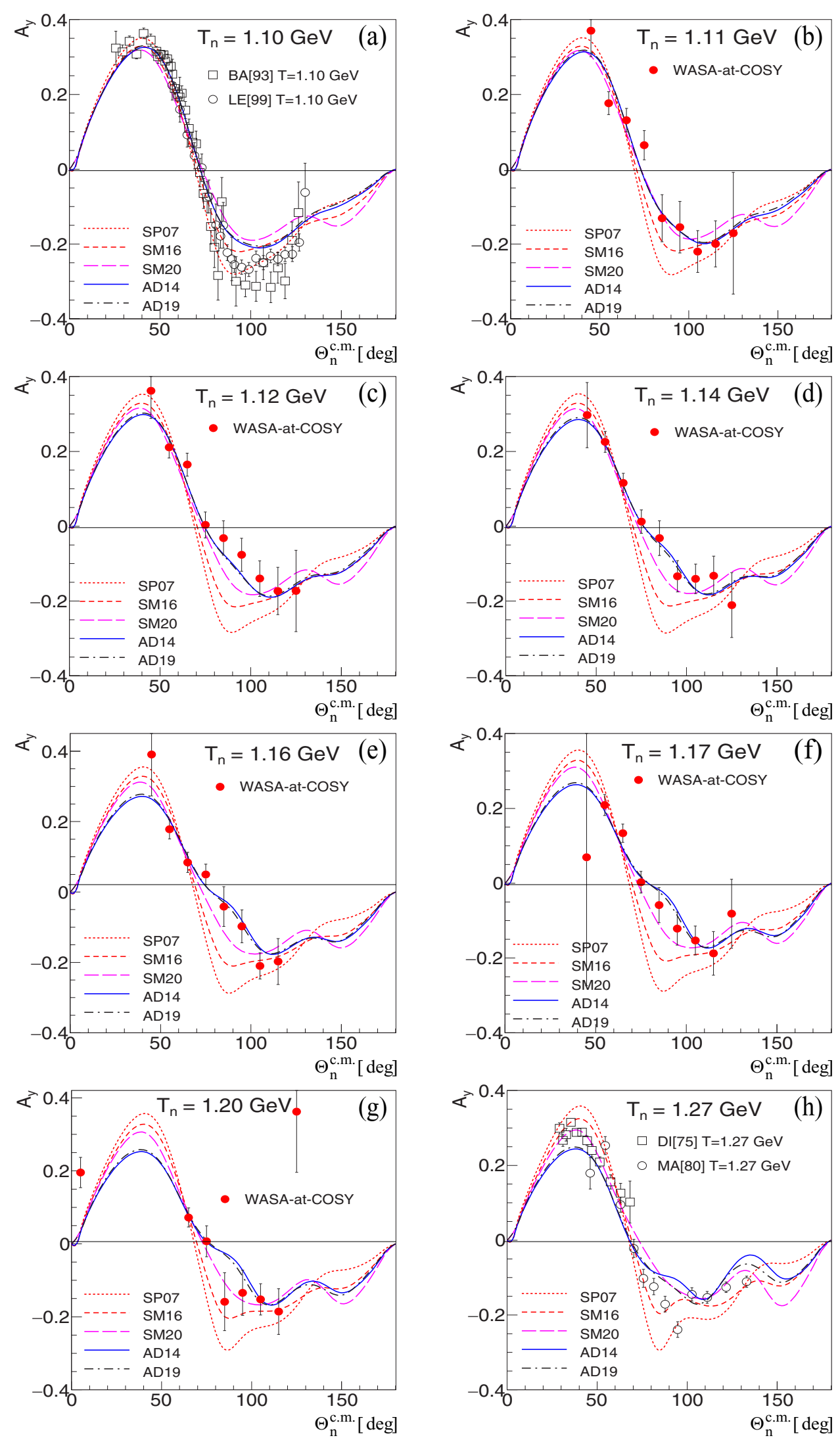

FIG. 3. Same as Fig. 2, but for angular distributions of the analyzing power. Filled symbols denote results from WASA-at-COSY [1,2]. Open symbols refer to previous measurements: for "BA[93]" see Ref. [31], for "LE[99]" see Ref. [32], for "DI[75]" see Ref. [33], and for "MA[80]" see Ref. [34]. 

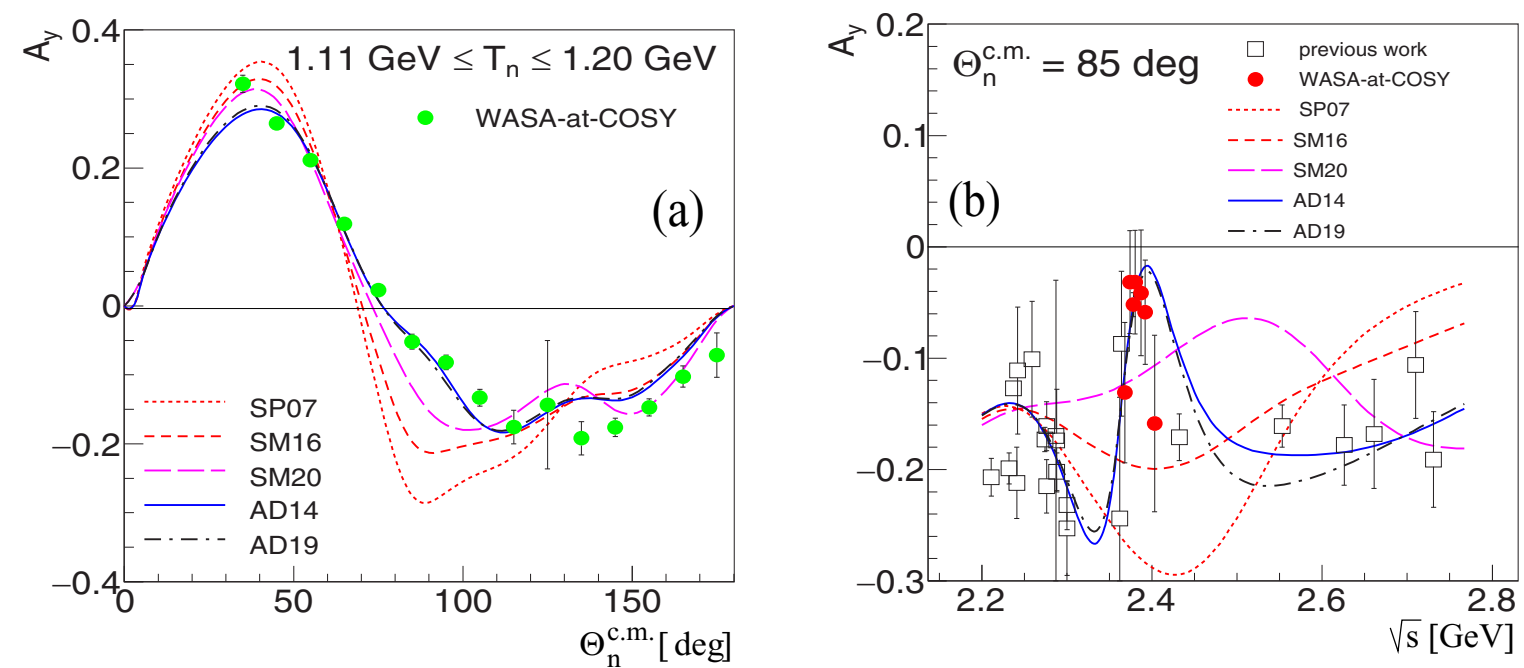

FIG. 4. Same as Fig. 3, but for (a) the angular distribution of the analyzing power averaged over the resonance region $\left(1.11 \mathrm{GeV} \leqslant T_{n} \leqslant\right.$ $1.20 \mathrm{GeV}$ ) and (b) the energy dependence of the analyzing power at $\Theta_{n}^{\mathrm{c} . \mathrm{m} .}=85^{\circ}$. Filled circles denote the WASA results [1,2]; open squares represent results from previous experiments [31-39].

scattering data available until 2007. The short-dashed curves represent the solution SM16 [26], which, in addition, is based on forward-angle $p p$-scattering data from COSY-ANKE. Both solutions exclude the pole of $d^{*}(2380)$ and hence do not provide a good description for the $n p$ analyzing power data $[1,2]$ measured by WASA-at-COSY in the region of the $d^{*}(2380)$ resonance as depicted in Fig. 3. These analyzingpower data, however, were included in the solution AD14, resulting in a resonance pole for $d^{*}(2380)$ in the coupled ${ }^{3} D_{3}-{ }^{3} G_{3}$ PWs $[1,2,27]$. This solution is denoted by solid lines in Figs. 2 and 3.

Whereas the SP07 and SM16 solutions give very similar results and provide only a qualitative description of the differential cross-section data, the AD14 solution successfully describes these data quantitatively, with the exception of the Birmingham data at $T_{p}=0.991 \mathrm{GeV}$ [22] and the back-angle data of Bizard et al. at $T_{p}=1.252 \mathrm{GeV}$ [17].

\section{PARTIAL-WAVE ANALYSES INCLUDING THE NEW CROSS-SECTION DATA}

The WASA-at-COSY cross-section data were also included in the GWU/SAID database for a new PW analysis. Since the AD14 solution already provides a quantitative description for the new cross-section data, it is no surprise that the inclusion of these data in the new PW analysis has no big impact and hence the resulting new solution AD19 (dash-dotted lines) is only marginally different from the AD14 solution. The small differences between the AD14 and the AD19 solutions may serve as a measure of the uncertainties in these solutions.

In order to check the uniqueness of these solutions many fits were carried out with varying initial weights for different data sets and other starting conditions. In this attempt, indeed a solution SM20 (long-dashed lines) was found, which comes closer to the analyzing power data than the previous solutions SP07 and SM16 did-as depicted in Fig. 3. However, this solution does much worse than AD14 and AD19 for the differential cross sections; see Fig. 2, where SM20 appears to be very close to the SP07 and SM16 results.

In order to investigate the SM20 solution in more detail, we compare the various GWU/SAID solutions in Fig. 4 with the WASA high-statistics data for the angular distribution of the analyzing power in the $d^{*}(2380)$ region [Fig. 4(a)] as well as with the energy dependence of the analyzing power near $90^{\circ}$ [Fig. 4(b)]. As pointed out in Refs. [1,2], the contribution of $d^{*}(2380)$ in the analyzing power is proportional to the associated Legendre function $P_{3}^{1}\left(\cos \Theta_{n}^{\text {c.m. }}\right)$. Therefore the resonance effect is at its maximum in the $90^{\circ}$ region; however, due to the richer database at $85^{\circ}$, Fig. 4(b) shows instead the energy dependence at that angle.

The WASA high-statistics data shown in Fig. 4(a) were obtained by not accounting for the spectator momentum. Thus these data represent a weighted average over the measured interval $\sqrt{s}=2.37-2.40 \mathrm{GeV}\left(T_{n}=1.11-1.20 \mathrm{GeV}\right)$ with a centroid at $\sqrt{s}=2.38 \mathrm{GeV}$; see Fig. 1 in Ref. [1] and Fig. 4 in Ref. [2], respectively. We see that for the various $\mathrm{PW}$ solutions the most critical angular region is around $90^{\circ}$, i.e., exactly the region that is most sensitive to the $d^{*}(2380)$ resonance. Whereas the $\mathrm{AD} 14$ and $\mathrm{AD} 19$ solutions reproduce the experimental data very well in this region, the other solutions miss the data there. Most striking is the failure of SP07. SM16 does a bit better and SM20 comes still closer but, nevertheless, fails quantitatively in this angular region.

In Fig. 4(a) we explore the energy dependence of the analyzing power in this angular region. There the data exhibit a pronounced pattern resembling the interference of a narrow resonance with the background. The solutions AD14 and AD19 are able to reproduce this pattern quantitatively, though the data suggest a somewhat narrower resonance pattern at 
the high-energy side. ${ }^{1}$ The SP07 solution predicts a smoothly curved energy dependence, which is far off the data, whereas the SM16 and SM20 solutions exhibit a somewhat flatter energy dependence, thus coming closer to the data on average, but still severely miss the resonance structure in the energy region of $d^{*}(2380)$.

We conclude that the solutions SP07, SM16, and SM20 all fail in a quantitative description of both cross-section and analyzing-power data in the energy region of $d^{*}(2380)$, whereas the solutions AD14 and AD19 quantitatively account for all experimental data.

In Fig. 5 we plot the ${ }^{3} D_{3}$ and ${ }^{3} G_{3}$ partial-wave amplitudes as well as their mixing term $\epsilon_{3}$ in dependence of the centerof-mass energy $W=\sqrt{s}$ for the solutions AD14 (solid curve), AD19 (dash-dotted curve), and SM20 (dashed curve). The amplitudes for the AD14 and AD19 solutions are very similar and differ slightly only at high energies. Both solutions exhibit a clear resonance structure in the $d^{*}(2380)$ region in both real and imaginary parts of all three amplitudes. In contrast, the SM20 solution exhibits only a very smooth energy dependence without an indication of any resonance.

In Fig. 6 we plot the Argand diagrams for the ${ }^{3} D_{3}$ [Fig. 6(a)] and ${ }^{3} G_{3}$ [Fig. 6(b)] PWs and their mixing amplitude $\epsilon_{3}$ [Fig. 6(c)] for the GWU/SAID solutions SM20 (dashed magenta curve), AD14 (solid blue curve), and AD19 (dash-dotted black curve). Whereas the SM20 solution shows no obvious looping in these diagrams, i.e., no sign of a pole, the solutions AD14 and AD19, which nearly coincide, do exhibit pronounced loops in accordance with the presence of the $d^{*}(2380)$ pole.

\section{PARTIAL-WAVE ANALYSIS AND DATA INTERPRETATION}

In the following, the search for poles presented in Ref. [2], based on analyzing Argand diagrams and the speed plot, is improved. Namely, a looping in the Argand diagram is, in the mathematical sense, only a necessary condition for the existence of a pole, and not yet a sufficient one. This simply means that, if a function has a pole, it must produce a counterclockwise looping, however, a counterclockwise looping found in Argand diagrams can be produced also by other effects, e.g., by branch points caused by channel openingsin our case by the nearby $N N^{*}(1440)$ threshold. To prove definitely that we indeed have a pole we need a more stringent method. Therefore, instead of analyzing Argand diagrams of ${ }^{3} D_{3},{ }^{3} G_{3}$, and the mixing term $\epsilon_{3}$ we introduce the trace of the ${ }^{3} D_{3}-{ }^{3} G_{3}$ matrix, and instead of quantifying the ${ }^{3} D_{3}$ partial wave with the speed-plot technique we quantify the whole trace with the Laurent + Pietarinen $(\mathrm{L}+\mathrm{P})$ expansion.

The coupled PWs in question create the following $I\left(J^{P}\right)=$ $0\left(3^{+}\right)$mixing matrix:

$$
T=\left[\begin{array}{cc}
{ }^{3} D_{3} & \epsilon_{3} \\
\epsilon_{3} & { }^{3} G_{3}
\end{array}\right]
$$

\footnotetext{
${ }^{1}$ This would be in accord with a somewhat narrower width of the resonance, as is in fact observed in the $N N \rightarrow N N \pi \pi$ channels [4].
}

Without loss of generality this matrix can be represented by its Laurent decomposition in its area of convergence:

$$
\begin{aligned}
T & =\left[\begin{array}{ll}
\frac{a_{11}+i b_{11}}{\text { Den }}+B_{11} & \frac{a_{12}+i b_{12}}{\text { Den }}+B_{12} \\
\frac{a_{12}+i b_{12}}{\text { Den }}+B_{12} & \frac{a_{22}+i b_{22}}{\text { Den }}+B_{22}
\end{array}\right], \\
\text { Den } & =M-W-i \Gamma .
\end{aligned}
$$

The ${ }^{3} D_{3}$ and ${ }^{3} G_{3}$ partial waves and the mixing term $\epsilon_{3}$ are shown in Fig. 5 for the GWU/SAID solutions SM20 (dashed magenta curve), AD14 (solid blue curve), and AD19 (dashdotted black curve).

Following the idea presented in Ref. [28] we use the trace ${ }^{2}$ of the $I\left(J^{P}\right)=0\left(3^{+}\right)$mixing matrix:

$$
\operatorname{Trace}[\mathrm{T}]=\frac{\left(a_{11}+a_{22}\right)+i\left(b_{11}+b_{22}\right)}{\operatorname{Den}}+\left(B_{11}+B_{22}\right) \text {. }
$$

As shown in Ref. [28], structures that are buried under notable background in individual matrix elements become evident once the trace of the matrix is viewed. In Fig. 7 we show the trace of all three GWU/SAID solutions.

The quantitative evaluation of the pole parameters of the Trace [T] was performed using the $\mathrm{L}+\mathrm{P}$ formalism, in a manner identical to that used in Ref. [29]. For the convenience of the reader let us repeat some basic facts.

The driving concept behind the method is to replace the complexity of solving an elaborate theoretical model and analytically continuing its solution into the complex energy plane by using a local power-series representation of PW amplitudes that exploit analyticity and unitarity. The $\mathrm{L}+\mathrm{P}$ approach separates the pole and regular part in the form of a Laurent expansion, and instead of modeling the regular part using some physical model it uses a conformal-mappinggenerated, rapidly converging power series with well-defined analytic properties called the Pietarinen expansion to represent it effectively. In other words, the method replaces the regular part calculated in a model with the simplest analytic function that has correct analytic properties of the analyzed PW (multipole) and fits the given input. In this approach the model dependence is minimized and is reduced to the choice of the number and location of $\mathrm{L}+\mathrm{P}$ branch points used in the model.

So, we expand the trace in terms of a sum over all poles and with a Pietarinen series representing the energy-dependent regular (nonpole) part as

$$
\text { Trace }[\mathrm{T}]=\sum_{i=1}^{k} \frac{\alpha_{-1}^{(i)}}{W-W_{i}}+B^{L}(W)
$$

Here $W, \alpha_{-1}^{(i)}$, and $W_{i}$ are complex numbers representing the c.m. energy, residues, and pole positions for the $i$ th pole, respectively, and $B^{L}(\mathrm{~W})$ is a regular function in the whole complex plane. As shown in Ref. [30], the generally unknown analytic function $B(W)$ with branch points in $x_{P}, x_{Q}$, and $x_{R}$

\footnotetext{
${ }^{2}$ The trace of the matrix is defined as the sum of its diagonal matrix elements, and due to its commutativity as $\operatorname{Trace}(\mathbf{A} \cdot \mathbf{B})=\operatorname{Trace}(\mathbf{B}$. A) it is identical for all matrices obtained from the original matrix by $U^{-1} \cdot \mathbf{A} \cdot U$ and, hence, for the diagonal one too.
} 

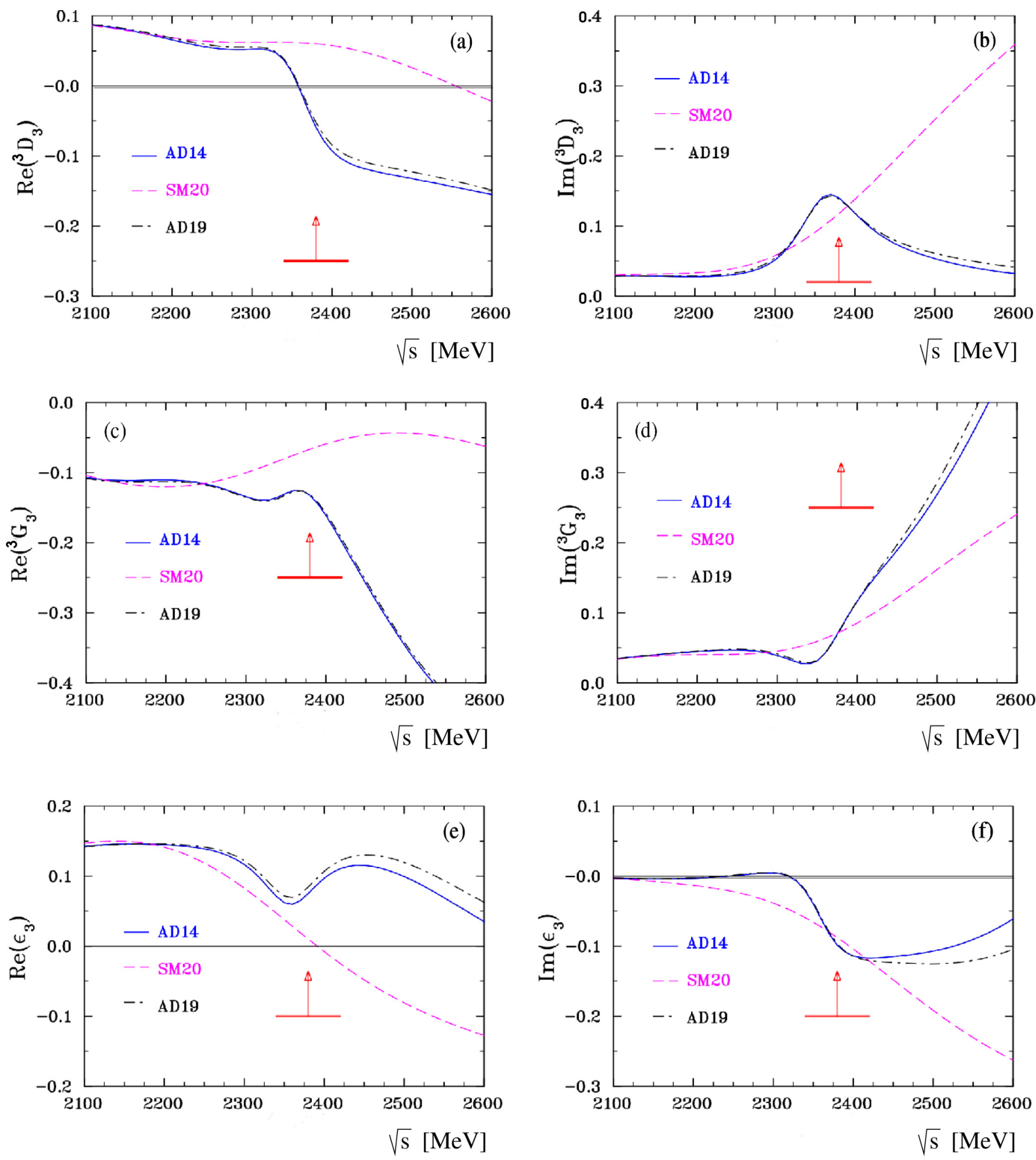

FIG. 5. (a), (c), (e) Real and (b), (d), (f) imaginary parts of the ${ }^{3} D_{3}$ (a), (b) and ${ }^{3} G_{3}$ (c), (d) partial-wave amplitudes and the mixing term $\epsilon_{3}$ (e), (f) for the GWU/SAID solutions SM20 (dashed magenta curve), AD14 (solid blue curve), and AD19 (dash-dotted black curve). Vertical arrows with horizontal bars indicate the location of mass $(m=2380 \mathrm{MeV})$ and width $(\Gamma=80 \mathrm{MeV})$ of $d^{*}(2380)$ as derived in Refs. [1,2]. 

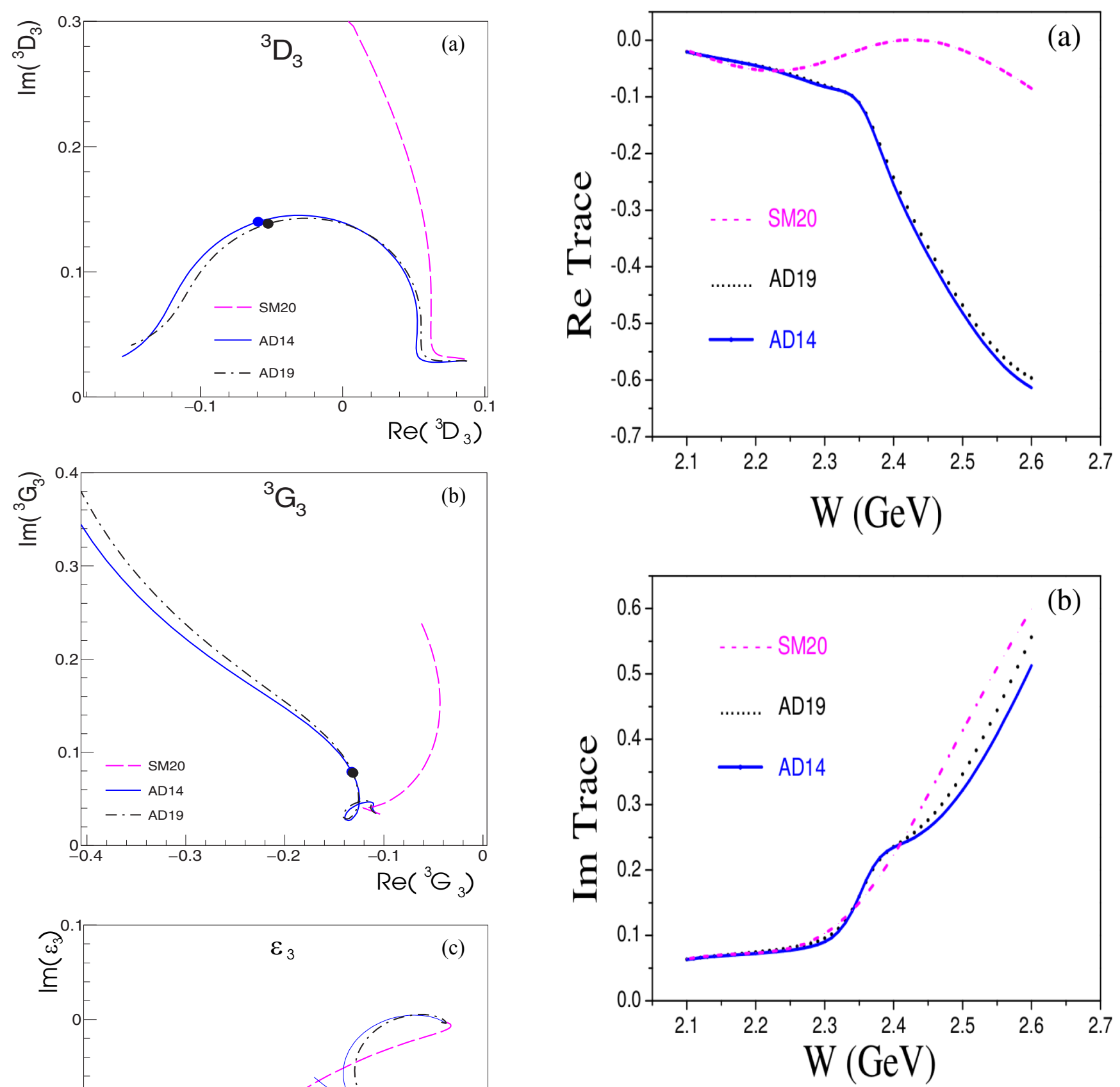

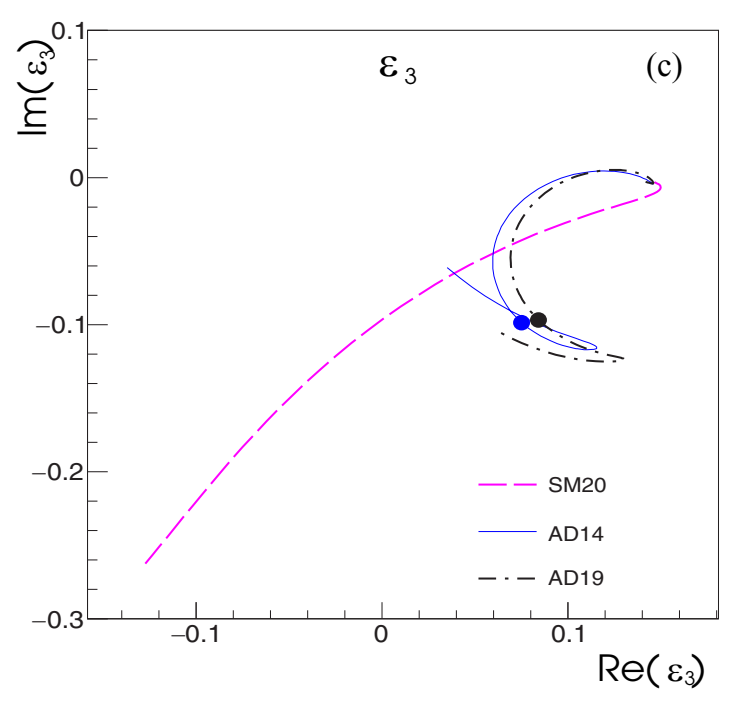

FIG. 6. Argand diagrams for (a) the ${ }^{3} D_{3}$ and (b) the ${ }^{3} G_{3}$ partialwave amplitudes and (c) their mixing term $\epsilon_{3}$ for the GWU/SAID solutions SM20 (dashed magenta curve), AD14 (solid blue curve), and AD19 (dash-dotted black curve). Small filled circles display the $d^{*}(2380)$ pole position.
FIG. 7. Trace of all three GWU/SAID partial-wave solutions: SM20 (dashed magenta curve), AD14 (solid blue curve), and AD19 (dotted black curve).

can be expanded into a power series of Pietarinen functions as

$$
\begin{aligned}
B^{L}(W)= & \sum_{n=0}^{M} c_{n} X(W)^{n}+\sum_{n=0}^{N} d_{n} Y(W)^{n} \\
& +\sum_{n=0}^{N} e_{n} Z(W)^{n}+\cdots, \\
X(W)= & \frac{\alpha-\sqrt{x_{P}-W}}{\alpha+\sqrt{x_{P}-W}}
\end{aligned}
$$


TABLE I. Results for resonance poles and branch points (bp) for the ideal case with errors of $0.1 \%$. Values in parentheses denote estimated uncertainties.

\begin{tabular}{lcccc}
\hline \hline & & SM20 & AD19 & AD14 \\
\hline & Real bp & $59.5(2)$ & $263.7(41.4)$ & $435(314)$ \\
$\chi_{\mathrm{df}}^{2}$ & Complex bp & $1.1(0.7)$ & $10.97(0.6)$ & $8.8(0.7)$ \\
& Real bp +1 pole & & $2.34(0.3)$ & $2.0(0.4)$ \\
Resonance in & Real bp & & $2352(1)-i 54(2)$ & $2348(1)-i 48(2)$ \\
3-body & Complex bp & $2260(22)-i 64(44)$ & & \\
subsystem & Real bp +1 pole & & & $2362(0.6)-i 109(2)$ \\
Genuine & Real bp & & & $2362(0.7)-i 114(2)$ \\
2-body & Complex bp & & & \\
resonance & Real bp +1 pole & & & \\
\hline \hline
\end{tabular}

$$
\begin{aligned}
& Y(W)=\frac{\beta-\sqrt{x_{Q}-W}}{\beta+\sqrt{x_{Q}-W}}, \\
& Z(W)=\frac{\gamma-\sqrt{x_{R}-W}}{\gamma+\sqrt{x_{R}-W}},
\end{aligned}
$$

where $c_{n}, d_{n}$, and $e_{n}$ and $\alpha, \beta$, and $\gamma$ are real numbers that represent tuning parameters and coefficients of the Pietarinen functions $X(W), Y(W)$, and $Z(W)$, respectively. A variable number of coefficients in the three series in Eq. (6) was used, depending on the structure of the nonpole part of each amplitude.

As the nearby energy points of the input PW trace are correlated through analyticity of the energy-dependent PW of the GWU/SAID solutions, the standard error analysis cannot be used, as the standardly defined $\chi^{2}$ becomes extremely small $\left(\chi^{2} \ll 1\right)$ regardless which error is attributed to the input. The method used is identical to what was done in Ref. [29] and is based on randomizing the central values of the energy-dependent solution with the partial-wave standard deviation $\sigma_{\mathrm{PW}}$ and assigning the error of the source energydependent point as the error of the randomized point. In this way we generate 1000 different sets, which are analyzed by $\mathrm{L}+\mathrm{P}$, and the error analysis is done in a standard way for noncorrelated quantities.

It is important to stress that our central problem is to establish whether or not the analyzed GWU/SAID solutions contain a pole. The $\mathrm{L}+\mathrm{P}$ approach by construction detects resonances in two ways: either as a resonance in a two-body process, which manifests itself as a pole on the real axes, or as a resonance in the three-body subsystem, which manifests itself as a complex branch point. In either of the two cases we encounter a resonance; however, there is still the matter of identifying its location, with the purpose of its identification.

The difference between the two situations is subtle. If we have a genuine pole in the two-body system, our real and imaginary parts will show a typical resonance behavior, and real branch points that represent the opening of two-body channels consisting of two stable particles should in principle be clearly visible, as they produce sharp cusps in the analyzed amplitude. However, where the branch point represents a channel which consists of a stable particle and a two-body resonant state, this branch point will become complex and the sharp cusp effect disappears. These two processes are different, but the method will require a much higher precision of the data to distinguish between the two. It is clear from Fig. 7 that our process will strongly depend on the confidence limit of all obtained GWU/SAID solutions. Namely, in the ideal case, when the confidence limit is high and the error band is low, the method will be able to distinguish between the two. However, as soon as the error bands become realistic, the clearly visible peak in the imaginary part will be smeared and the distinction between the two scenarios (genuine pole and complex branch point) will be lost.

Therefore, we produce three sets of results: solution (a), given in Table I with an estimated error of $0.1 \%$; solution (b), with the error increased by five times, to $0.5 \%$; and (c) in Table II with the realistic error of the GWU/SAID solutions estimated to be $2 \%$.

What we immediately see in the tables is that the clarity of the effect is greater the smaller the error bars are, and this is what we expected. A pole is certainly detected for the AD14 and AD19 solutions, but it is not clear whether it is a real two-body resonance in the two-body system materialized as a genuine pole or a two-body resonance in the three-body subsystem materialized as a complex branch point. For the third SM20 solution the possibility of a pole in the form of a complex branch point is preferred only for ideal cases with unrealistically small error bars, but for realistic error bars the situation is ambiguous. For the smallest error bars in Table I we see that all three solutions including SM20 require at least a complex branch point, but for the realistic error in Table II it is only clear that real branch points are much less likely for all three solutions ( $\chi_{\mathrm{df}}^{2}$ is the largest, but not convincingly). On the other hand, for the ideal case given in Table I it is very likely that these results could be interpreted only as a resonance in the two-body system, as $\chi_{\mathrm{df}}^{2}$ is notably higher for the other two possibilities-real and complex branch points. Our test with errors of $0.5 \%$ shows that already in this case an equal probability for the real pole and the complex branchpoint solutions is reached.

Therefore, we may conclude the following. Both AD solutions require a pole in the system, however, the distinction between a pole in the two-body system and one in the three- 
TABLE II. Same as Table I, but for the realistic error of $2 \%$.

\begin{tabular}{lcccc}
\hline \hline & & SM20 & AD19 & AD14 \\
\hline & Real bp & $1.38(0.3)$ & $1.83(0.2)$ & $2.06(0.3)$ \\
$\chi_{\mathrm{df}}^{2}$ & Complex bp & $0.97(0.16)$ & $0.99(0.16)$ & $0.98(0.16)$ \\
& 1 pole & & $1.03(0.15)$ & $1.08(0.5)$ \\
Resonance in & Real bp & & $2361(14)-i 59(21)$ & $2354(12)-i 44(20)$ \\
3-body & Complex bp & $2265(76)-i 0(7)$ & & $2361(11)-i 60(13)$ \\
subsystem & Real bp +1 pole & & & \\
Genuine & Real bp & & & $2361(21)-i 63(20)$ \\
2-body & Complex bp & & & 2 \\
resonance & Real bp +1 pole & & & \\
\hline \hline
\end{tabular}

body subsystem depends on the reliability of the GWU/SAID solutions. The numerical quantification also depends on the confidence limit of the GWU/SAID solutions.

However, if we add information from sources other than just elastic $p n$ scattering, then the complex branch-point solution can be safely discarded. As noted above, the only possible three-body branch point in the vicinity of the found pole location is due to the $N N^{*}(1440)$ configuration. The Roper resonance $N^{*}(1440)$ is much broader than suggested by the imaginary part of the pole given in Table II. And since it is formed near threshold in the isoscalar part of the $N N \rightarrow N N^{*}(1440)$ reaction preferentially by the ${ }^{3} S_{1} N N$ partial wave, a significant formation by the isoscalar ${ }^{3} D_{3}-{ }^{3} G_{3}$ partial waves appears very unlikely. Finally, $d^{*}$ (2380), which may be identified with the found pole, does not decay into $N N^{*}(1440)(\mathrm{BR}<14 \%$ at $90 \%$ C.L. $)$ according to the recent measurement of the isoscalar part of the $N N \rightarrow N N \pi$ reaction [10].

In our tests with various error assignments to the GWU/SAID solutions the location of the pole position appears to be remarkably stable against these error variations. The result of 2361(21) MeV is compatible with the traditional speed-plot result of 2380(10) $\mathrm{MeV}$ [2] within uncertainties as well as with the result from the $n p \rightarrow d \pi^{0} \pi^{0}$ reaction [4], where a value of $2.37 \mathrm{GeV}$ was observed for the $d^{*}(2380)$ resonance energy.

The situation with respect to the resonance width is more delicate. Though the value of $126(40) \mathrm{MeV}$ for the width deduced with the $\mathrm{L}+\mathrm{P}$ method still overlaps within uncertainties with that deduced by the speed-plot technique, 80(10) $\mathrm{MeV}$, it is at notable variance with the result of $70 \mathrm{MeV}$ from $n p \rightarrow d \pi^{0} \pi^{0}$. There are several reasons for this discrepancy. First, as noted in the discussion of the energy excitation function of the analyzing power around $90^{\circ}$ - where the resonance effect of $d^{*}(2380)$ is largest-the measured resonance structure on the high-energy side is narrower than accounted for by the AD solutions. This failure causes a long high-energy tail of the resonance structure seen in $\operatorname{Im}\left({ }^{3} D_{3}\right)$ [Fig. 5(b)]. Consequently, the resonance effect appears to be more extended in the PW solutions than in the data. Second, high-quality data beyond $\sqrt{s}=2.44 \mathrm{GeV}$ are rare in the GWU/SAID database and hence the uncertainties in the various PW solutions increase rapidly beyond this energy. That is, the high-energy tail of the $d^{*}(2380)$ resonance is not well fixed in the PW solutions, causing a large uncertainty in the separation of pole and background. This is particularly true for the L $+\mathrm{P}$ method, where the resonance shape is kept unconstrained as much as possible. Hence the true uncertainties for the imaginary part of the pole appear to be even larger than given in Table II.

\section{SUMMARY AND CONCLUSIONS}

New data for the differential cross sections in the energy region of the $d^{*}(2380)$ dibaryon resonance have been presented. They were extracted from exclusive and kinematically complete measurements of quasifree $\vec{n} p$ scattering using the WASA detector setup at COSY and having a polarized deuteron beam impinged on the hydrogen pellet target. The new cross-section data supplement the analyzing-power data published earlier [1,2].

The new cross-section data are at obvious variance with the GW/SAID partial-wave solutions SP07, SM16, and SM20, however, they agree quantitatively with the solutions AD14 and AD19. Whereas the first ones do not contain the $d^{*}(2380)$ pole, the latter two do include this pole. The solution AD14 was obtained in 2014 by inclusion of the WASA analyzingpower data in the SAID database. These data then produced the $d^{*}(2380)$ pole in the ${ }^{3} D_{3}-{ }^{3} G_{3}$ coupled PW. It is very gratifying and simultaneously demonstrates the predictive power of this solution that it is able to provide a quantitative description of the new data on the differential cross sections. The new solution AD19, which includes the new cross-section data, deviates from the AD14 solution only marginally.

Since a counterclockwise looping in the Argand diagram is a necessary, but not sufficient condition for a resonance pole, the three GWU/SAID solutions AD14, AD19, and SM20 were subjected to an interpretation within the Laurent + Pietarinen method. The conclusion there is that a pole at the position of the $d^{*}(2380)$ resonance is clearly confirmed. However, understanding the effect as a consequence of the $N N^{*}$ (1440) branch point in the three-body subchannel can be excluded definitively only by using additional information about the isoscalar part of the $N N \rightarrow N N \pi$ reaction. Based 
on the elastic pn scattering alone, a strict elimination of the branch-point interpretation would necessitate new precise high-quality measurements, in particular, at energies beyond $\sqrt{s}=2.4 \mathrm{GeV}$, in order to approach the precision given in Table I.

The value of 2361(21) MeV derived for the pole position of $d^{*}(2380)$ by the new $\mathrm{L}+\mathrm{P}$ method agrees very well with the values obtained previously [1,2,4-7]. But the value of 126(40) MeV for the width comes out too large in comparison with the value of $80(10) \mathrm{MeV}$ derived by the conventional method [1,2] as well as with the value of $70 \mathrm{MeV}$ observed directly in the data for the $N N \rightarrow N N \pi \pi$ channels [4-7]. The reason for this appears to be the lack of precision data for $n p$ scattering on the high-energy side of $d^{*}(2380)$ beyond $\sqrt{s}=2.4 \mathrm{GeV}$.

\section{ACKNOWLEDGMENTS}

We acknowledge valuable discussions with $\mathrm{C}$. Hanhart and C. Wilkin on this issue. This work was supported by BMBF, Forschungszentrum Jülich (COSY-FFE), and the German Research Foundation (DFG; CL214/3-2 and CL214/3-3). Three of us (H.Cl., I.S., and A.Š) appreciate the support by the Munich Institute for Astro- and Particle Physics (MIAPP), which is funded by the Deutsche Forschungsgemeinschaft (DFG) under Germany's Excellence Strategy-EXC-2094 (390783311). This work was supported (W.B., I.S., and R.W.) in part by the US Department of Energy, Office of Science, Office of Nuclear Physics, under Awards No. DE-SC0016583 and No. DE-SC0016582.
[1] P. Adlarson et al., Phys. Rev. Lett. 112, 202301 (2014).

[2] P. Adlarson et al., Phys. Rev. C 90, 035204 (2014).

[3] M. Bashkanov et al., Phys. Rev. Lett. 102, 052301 (2009).

[4] P. Adlarson et al., Phys. Rev. Lett. 106, 242302 (2011).

[5] P. Adlarson et al., Phys. Lett. B 721, 229 (2013).

[6] P. Adlarson et al., Phys. Rev. C 88, 055208 (2013).

[7] P. Adlarson et al., Phys. Lett. B 743, 325 (2015).

[8] G. Agakishiev et al., Phys. Lett. B 750, 184 (2015).

[9] H. Clement, M. Bashkanov, and T. Skorodko, Phys. Scripta T166, 014016 (2015).

[10] P. Adlarson et al., Phys. Lett. B 774, 599 (2017).

[11] M. Bashkanov, H. Clement, and T. Skorodko, Eur. Phys. J. A 51, 87 (2015).

[12] H. Clement, Prog. Part. Nucl. Phys. 93, 195 (2017).

[13] A. Gal, Phys. Lett. B 769, 436 (2017).

[14] T. Ishikawa et al., Phys. Lett. B 772, 398 (2017).

[15] M. S. Guenther (for the A2 Collaboration), PoS (HADRON2017), 051 (2017).

[16] Y. Terrien et al., Phys. Rev. Lett. 59, 1534 (1987).

[17] G. Bizard et al., Nucl. Phys. B 85, 14 (1975).

[18] J. Bystricky et al., J. Phys. 48, 1901 (1987).

[19] Chr. Bargholtz et al., Nucl. Inst. Methods A 594, 339 (2008).

[20] H. H. Adam et al., arXiv:nucl-ex/0411038.

[21] R. Machleidt, Phys. Rev. C 63, 024001 (2001).

[22] T. A. Murray et al., Nuovo Cimento 49, 261 (1969).

[23] M. L. Perl et al., Phys. Rev. D 1, 1857 (1970).
[24] SAID database, http://gwdac.phys.gwu.edu/

[25] R. A. Arndt, W. J. Briscoe, I. I. Strakovsky, and R. L. Workman, Phys. Rev. C 76, 025209 (2007).

[26] R. L. Workman, W. J. Briscoe, and I. I. Strakovsky, Phys. Rev. C 94, 065203 (2016).

[27] R. L. Workman, W. J. Briscoe, and I. I. Strakovsky, Phys. Rev. C 93, 045201 (2016).

[28] S. Ceci, A. Švarc, B. Zauner, D. M. Manley, and S. Capstick, Phys. Lett. B 659, 228 (2008).

[29] W. J. Briscoe et al., Phys. Rev. C 100, 065205 (2019).

[30] A. Švarc, M. Hadžimehmedović, H. Osmanović, J. Stahov, L. Tiator, and R. L. Workman, Phys. Rev. C 88, 035206 (2013).

[31] J. Ball et al., Nucl. Phys. A 559, 489 (1993).

[32] A. de Lesquen et al., Eur. Phys. J. C 11, 69 (1999).

[33] R. Diebold et al., Phys. Rev. Lett. 35, 632 (1975).

[34] Y. Makdisi, M. L. Marshak, B. Mossberg, E. A. Peterson, K. Ruddick, J. B. Roberts, and R. D. Klem, Phys. Rev. Lett. 45, 1529 (1980).

[35] C. R. Newsom et al., Phys. Rev. C 39, 965 (1989).

[36] J. Arnold et al., Eur. Phys. J. C 17, 67 (2000).

[37] J. Ball et al., Nucl. Phys. B 286, 635 (1987).

[38] M. W. McNaughton et al., Phys. Rev. C 48, 256 (1993); 53, 1092 (1996).

[39] G. Glass, T. S. Bhatia, J. C. Hiebert, R. A. Kenefick, S. Nath, L. C. Northcliffe, W. B. Tippens, and J. E. Simmons, Phys. Rev. C 47, 1369 (1993). 